\title{
Infrasonic Nanocrystal Formation in Amorphous NiTi Film: Physical Mechanism, Reasons and Conditions
}

\author{
Evgeny E. Slyadnikov
}

check for updates

Citation: Slyadnikov, E.E. Infrasonic Nanocrystal Formation in Amorphous NiTi Film: Physical Mechanism, Reasons and Conditions. Metals 2021, 11, 1390. https:// doi.org/10.3390/met11091390

Academic Editor: Giovanni Principi

Received: 8 August 2021

Accepted: 30 August 2021

Published: 1 September 2021

Publisher's Note: MDPI stays neutral with regard to jurisdictional claims in published maps and institutional affiliations.

Copyright: (C) 2021 by the author. Licensee MDPI, Basel, Switzerland. This article is an open access article distributed under the terms and conditions of the Creative Commons Attribution (CC BY) license (https:/ / creativecommons.org/licenses/by/ $4.0 /)$.
ISPMS Institute of Strength Physics and Material Science SB RAS, Akademicheskii pr. 2/4, 634055 Tomsk, Russia; eeslyadnikov@gmail.com; Tel.: +7-923-426-4969

\begin{abstract}
The physical mechanism, reasons and conditions of nanocrystal formation in an amorphous NiTi metal film, stimulated by infrasonic action, are formulated. Nanostructural elements of an amorphous medium (relaxation centers) containing disordered nanoregions with two-level systems are considered to be responsible for this process. When exposed to infrasound, a large number of two-level systems are excited, significantly contributing to inelastic deformation and the formation of nanocrystals. The physical mechanism of the nanocrystallization of metallic glass under mechanical action includes both local thermal fluctuations and the additional quantum tunneling of atoms stimulated by shear deformation. A crystalline nanocluster appears as a result of local atomic rearrangement growing increasingly exposed to infrasound. It is possibly unstable in the absence of infrasound. When the radius of the nanocluster reaches a critical value, a potential well appears, in which a conducting electron is localized to form a phason (stable nanocrystal). Estimated values of the phason's radius and the depth of the nanometer potential well is about $0.5 \mathrm{~nm}$ and $1 \mathrm{eV}$, respectively. It forms a condition of stable phason formation. The occurrence of the instability of the amorphous state and following transformation to the nanostructured state is based on the accumulation of the potential energy of inelastic deformation to a critical value equal to the latent heat of the transformation of the amorphous state into the nanostructured state.
\end{abstract}

Keywords: physical mechanism; nanocrystals; amorphous metal film; infrasound; inelastic deformation; quantum tunneling; localization of the conduction electron

\section{Introduction}

Amorphous metal alloys with a nanostructure are possessed by unique mechanical properties occurring due to intensive mechanical processing [1]. For nanostructuring an amorphous NiTi film, low-frequency mechanical vibrations are used at a temperature much lower than the glass transition temperature used for the remaining sample deformation within the elastic region [2-4]. Data obtained by the methods of X-ray diffraction and highresolution transmission electron microscopy indicate that the initial alloy is homogeneously amorphous, while the structure of the sample subjected to a mechanical vibration amplitude of $1 \mathrm{mkm}$ is close to the structure of the initially untreated sample (the short-range order changes and free volume decreases). In the structure of the sample subjected to mechanical vibrations with an amplitude of $4 \mathrm{mkm}$, nonspherical clusters with correct atomic positions and sizes of 3-5 $\mathrm{nm}$ appear in an amorphous matrix.

It has been established [2] that, under mechanical action, the latent heat of crystallization decreases slightly. It means that the free energy of the state with nanocrystals becomes less in comparison with the free energy of the amorphous state wherein the chemical and phase composition of alloys with and without nanocrystals is the same. Therefore, nanocrystallization caused by infrasonic stimulation is not related to diffusion, but provoked by collective atomic rearrangements during structural relaxation.

Further experimental studies have shown that [3] an increase in the duration of oscillations up to $2 \mathrm{~h}$ enlarges the size of clusters and their growth in different directions. 
Running on mechanical vibrations for $4 \mathrm{~h}$, the film acquires a crystalline structure. It consists of irregular shape grains of different crystallographic orientations with amorphous islands embedded.

In [4], the influence of temperature, frequency and amplitude of vibrations (the duration of vibrations is $10 \mathrm{~min}$ ) on the structure of amorphous alloys based on NiTi was investigated. It was found that, at room temperature, a vibration frequency of $20 \mathrm{~Hz}$, a vibration amplitude of $4 \mu \mathrm{m}\left(10^{-6} \mathrm{~m}\right)$, and crystalline clusters $4-6 \mathrm{~nm}\left(10^{-9} \mathrm{~m}\right)$ in size appear in the amorphous matrix. With an increase in the initial temperature of the sample (from 25 to $200{ }^{\circ} \mathrm{C}$ ), the distribution and size of crystalline clusters practically do not change. The crystallization temperature practically does not change, and the latent heat of crystallization decreases with an increase in the vibration amplitude and the initial temperature of the sample. When the frequency (below $5 \mathrm{~Hz}$ ) or the vibration amplitude (down to $1 \mu \mathrm{m}$ ) decreases, crystalline clusters do not appear in the amorphous matrix.

Despite the fact that the phenomenon of nanocrystal formation in amorphous NiTi film under the action of mechanical vibrations is described in the literature, the physical mechanisms, reasons and conditions of its occurrence remain unclear.

In accordance with classical concepts [5], the nucleation of crystals in amorphous alloys can occur due to either a homogeneous or heterogeneous mechanism. Approaching the glass transition temperature, the homogeneous nucleation occurs through the fluctuation nucleus formation with a radius larger than the critical one [5]. Heterogeneous nucleation occurs mainly at temperatures below the glass transition temperature placed on defects, boundaries, etc., $[5,6]$ and due to "frozen in" crystallization centers [7]. The density of heterogeneous nuclei in metal melts is about $10^{11}-10^{12} \mathrm{~m}^{-3}$. Amorphous alloys, without nanosized crystallites, have a density noticeably exceeding these values (for example, in the $\mathrm{Fe}_{80} \mathrm{~B}_{20}$ alloy, the density of crystallites is $10^{18} \mathrm{~m}^{-3}$ [8]). In nanostructured systems (for example, in Cu-Ti alloys [9]), the density of crystallites can reach $10^{25} \mathrm{~m}^{-3}$; therefore, most of the nuclei arise through homogeneous nucleation during annealing.

As analysis shows, the mechanisms of the nanocrystal formation in amorphous NiTi film under stationary annealing and mechanical action differ significantly. During thermal annealing, the sample temperature is much higher than room temperature. The nanocrystallization process is slow and homogeneous. Nanocrystals appear throughout the entire volume by the thermal fluctuation mechanism. Under mechanical action, the medium temperature is room temperature; therefore, the probability of nanocrystal formation by the usual thermal fluctuation mechanism is much less than at the annealing temperature. However, during mechanical vibrations with an amplitude of $4 \mathrm{mkm}$, significant shear stresses arise in the film. At a critical value of shear stress, the medium loses shear stability, and inelastic deformation occurs. Therefore, the mechanism of nanocrystallization of metallic glass under mechanical action should include quantum tunneling of atoms or atomic groups [10-14] stimulated by shear deformation in addition to local thermal fluctuations.

The ability of amorphous metal alloys to cause irreversible deformation and nanocrystal formation is associated with the collectivized character of the metallic interatomic bond [15]. Irreversible processes of local collective atomic rearrangements can occur much more easily in the presence of conducting electrons. Therefore, when constructing the mechanism of the irreversible deformation and nanocrystallization of amorphous alloys, it is necessary to take into account the influence of the electronic subsystem [16-18]. Specifically, for metallic glass NiTi, it is necessary to consider the localization of a conducting electron in a nanometer potential well and the formation of a phason [18].

The study of nanocrystal formation under a different external surrounding is of interest to the modern science of solid state matter. At the same time, as we found in the literature, there is no consideration of the effect of infrasound nanocrystal formation, and its physical mechanisms and possible conditions are not established.

The present work aims to consider the physical mechanisms of nanocrystal formation in amorphous NiTi under the infrasound action. The mechanism of influence of infrasound is not known and has not been previously considered. Herein, we propose a model that 
takes into account the inhomogeneity of the amorphous medium, the so-called relaxation centers, capable of rearranging according to the mechanism of athermal shear deformations stimulated by infrasound. As a result of the atomic rearrangement of the relaxation center, a more ordered structure is formed. As a consequence, a potential well arises, in which a conductivity electron is localized.

As we supposed, the analytical formulation of the problem is of two objectives: (1) in the framework of the model to formulate the equations of the density operator, taking into account the relaxation term and the field of static shear deformation; (2) to determine the probabilities of transitions of an atom in a double-well potential by thermal and athermal, i.e., quantum, mechanisms.

At the same time, numerical estimations might be provided in order to make the process of crystallization predictable. (3) It is likely to know the value of the shear strain, epsilon, at which quantum tunneling (disordered nanocluster transforms to a nanocrystal) occurs according to the athermal type; (4) It is necessary to determine conditions for the appearance of a localized state of conductivity electron at the potential well of the nanocrystal. There is a need in quantitative estimations of the critical values of the potential well depth and the radius of a stable nanocrystal.

\section{Physical Mechanism of Inelastic Deformation of Metal Glasses}

Inelastic deformation occurring as a response to the applied shear stress [19-24] is explained by local static displacements of a group of atoms (or an atom) from the initial equilibrium positions to the new ones at a distance less than interatomic spacing.

The first microscopic model of elementary cooperative inelastic rearrangements in metallic glasses is based on the concept of a uniformly distributed free volume. It was proposed by Argon in 1979 [19]. In the model [20], it is assumed that relaxation centers appear due to the inhomogeneity of the glass structure, particularly in the regions with an excess free volume relative to the "ideal structure". At present, the model of the activation energies spectrum [21], as well as the model of directed structural relaxation oriented by an external force [22], is often used.

The model [23] proposes a method of description of the local structure of amorphous alloys on the basis of the concept of $n, p$, and $\tau$ defects. In [24], a polycluster model of the structure of an amorphous state was proposed. The elementary rearrangements of atomic configurations occur at intercluster boundaries under the action of external forces. At high temperatures, the rearrangements of configurations are, possibly, a mechanism of thermal fluctuations, whereas at low temperatures quantum tunneling is more physically suitable.

The structural elements of an amorphous medium containing free volume (relaxation centers [20]) rearranging not only as a result of thermal fluctuations but due to quantum tunneling are of particular interest. Atom moving in a double-well potential (Figure 1) or a group of atoms with two configurations possessing different energies considered as twolevel systems [10-14,24]. The atom transition from one well to another (or the changing of one configuration of atoms to another), which means a transition from one energy level to another, can occur due to quantum tunneling. These effects are more probable when a two-level system is close to degenerating. Specifically, the levels of zero-point vibrations of an atom, in each of the neighboring wells, should differ slightly without taking into account tunneling, (i.e., there is a resonance detuning $\Delta_{0}$ ). A two-level system is characterized by an initial resonance detuning $\Delta_{0}<<1 \mathrm{eV}$ and an initial frequency of tunnel junctions $\omega_{K}^{0}=\omega_{0} \exp \left(-\frac{a}{a_{d B}}\right)$. Here, $a_{d B}$ is the de Broglie wavelength and $a$ is the width of the potential barrier in the double-well potential, $\omega_{0} \sim 10^{13} \mathrm{~Hz}$. The frequency, $\omega_{K}^{0}$, on the one hand, must be much lower $\Delta_{0}$ in order for the initial splitting of the levels $E_{0}=\sqrt{\Delta_{0}^{2}+\hbar^{2}\left(\omega_{K}^{0}\right)^{2}}$ to be small $\sim \Delta_{0}$. On the other hand, $\omega_{K}^{0}$ should not be too small for the tunneling to manifest itself during experiment. 


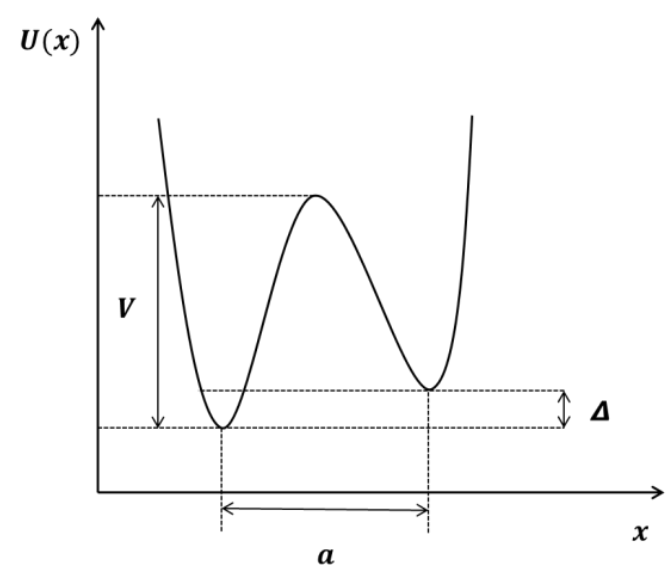

Figure 1. Dependence of the double-well potential $U(x)$ on the spatial coordinate $x$. $\Delta$-resonance detuning; $V$-interwell barrier; $a$-width of the potential barrier.

In the framework of the one-particle model of the interaction of phonons with twolevel systems [10-14,24], the initial resonance detuning $\Delta_{0}$ and the initial frequency of tunneling transitions $\omega_{K}^{0}$ are renormalized. During the passage of a sound wave, the atoms that form the potential curve of the two-level system are displaced. As a consequence, individual wells of the curve are distorted, which leads to a change in the resonance detuning $\Delta$. The shape of the potential barrier also changes and shifts to the frequency of tunnel junctions $\omega_{K}$. The distance between the two levels of the two-level system becomes to be equal to $E=\sqrt{\Delta^{2}+\hbar^{2} \omega_{K}^{2}}$. For the linear approximation of deformation, we have

$$
\delta \Delta=\sum_{i, j} B_{i j} \varepsilon_{j i}, \delta \omega_{K}=\sum_{i, j} D_{i j} \varepsilon_{j i}, \varepsilon_{i j}=\frac{1}{2}\left(\frac{\partial u_{i}}{\partial x_{j}}-\frac{\partial u_{j}}{\partial x_{i}}\right)
$$

Here, $B_{i j}, D_{i j}$ are tensors of strain potentials, which depend on the local structure of the glass in the place where the two-level system is located. $\varepsilon_{i j}$-strain tensor, which is expressed through the components of the displacement vector $u_{i}$ The energy per atom per one deformation cycle is $e_{a} \cong 10^{-6} \mathrm{eV}$; therefore, $\delta \Delta \cong e_{a} \cong 10^{-6} \mathrm{eV}$. Since at the initial moment of time $\Delta_{0}<0$, it is possible to estimate $\left|\Delta_{0}\right| \cong \delta \Delta \cong 10^{-6} \mathrm{eV}$ and the maximum value of $\omega_{K} \cong 10^{9} \mathrm{~s}^{-1}$.

To estimate the characteristic parameters of the atomic tunneling, we will perform a modeling calculation. Let us suppose an atom in the relaxation center is in a two-well potential or there is a group of atoms in which there are two configurations with slightly different energies [12-14,24]. The potential energy of such a subsystem can be represented as the sum of two single-well potentials $U_{L}$ and $U_{R}$. At the initial moment of time (in the absence of mechanical load), the left and right potential holes have different depths-the left hole is deeper than the right one. The action of an external mechanical force on the system leads to a change in the distances between the atoms of the medium (deformation), which in turn changes the shape of the double-well potential.

Let the wave functions $\Psi_{L}, \Psi_{R}$ and energy eigenvalues $E_{L}^{0}, E_{R}^{0}$ be known for the Schrödinger equation with single-well potentials $U_{L}, U_{R}$. For simplicity, when superimposing the field of the static shear component of deformation $\varepsilon$, only the energy eigenvalues $E_{L}^{0}, E_{R}^{0}$ are renormalized, according to the rule $E_{L}=E_{L}^{0}+B \varepsilon, E_{R}=E_{R}^{0}-B \varepsilon$. The complete Hamiltonian of a one-dimensional subsystem with a two-well potential $U_{L}(\varepsilon)+U_{R}(\varepsilon)$, $U_{L}(\varepsilon)=U_{L}+B \varepsilon, U_{R}(\varepsilon)=U_{R}-B \varepsilon$ in the field of a static shear strain component $\varepsilon$ has the form.

$$
H=\frac{-\hbar^{2}}{2 m} \frac{\partial^{2}}{\partial x^{2}}+U_{L}(\varepsilon)+U_{R}(\varepsilon), \Psi=a_{L} \Psi_{L}+a_{R} \Psi_{R}, \Delta=E_{L}-E_{R}=E_{L}^{0}-E_{R}^{0}+2 B \varepsilon
$$


Due to the assumed weak overlap of wave functions $\Psi_{L}, \Psi_{R},\left(S=\int d x \Psi_{L} \Psi_{R} \ll 1\right)$, solving the Schrödinger equation with Hamiltonian (2), we find the stationary levels $E_{ \pm}$(Figure 2) and stationary states $\Psi_{ \pm}$, in which an atom is delocalized between two wells:

$$
\begin{gathered}
E_{ \pm}=\frac{1}{2}\left[E_{L}+E_{R} \pm \sqrt{D}\right], D=\Delta^{2}+4 U_{L R}^{L} U_{L R}^{R}, U_{L R}^{L}=\int d x \Psi_{L} U_{L}(\varepsilon) \Psi_{R} \\
\Psi_{+}=[\Delta+\sqrt{D}] \Psi_{L}+2 U_{L R}^{R} \Psi_{R}, \Psi_{-}=2 U_{L R}^{L} \Psi_{L}-[\Delta+\sqrt{D}] \Psi_{R}
\end{gathered}
$$

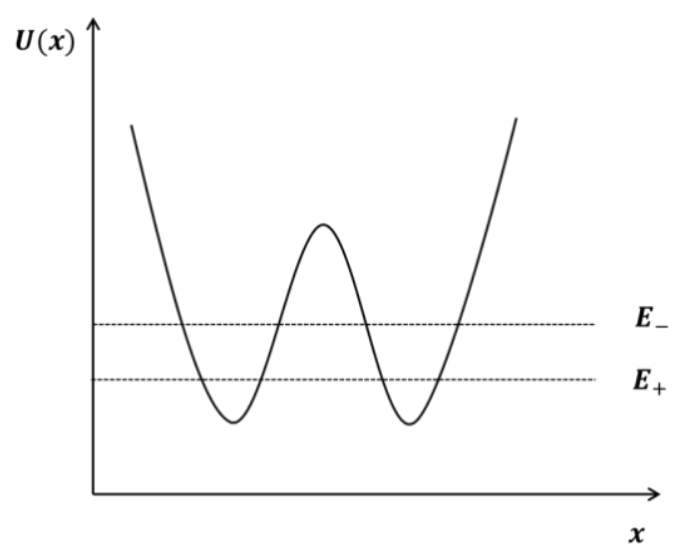

Figure 2. Ground state for a symmetric $(\Delta=0)$ double-well potential $U(x)$. Quantum tunneling removes the initial degeneracy of the levels. $E_{+}$- energy eigenvalue for a symmetric wave function $\Psi_{+} ; E_{-}-$energy eigenvalue for an antisymmetric wave function $\Psi_{-}$.

Let us investigate the tunneling transition of an atom from one potential well to another in the field of the static shear component of deformation $\varepsilon$ at a finite temperature using the formalism of the density operator $\rho$ [25]. In this case, the atom making transitions between the wells interacts with the thermostat. Using eigenvectors $\Psi_{L}, \Psi_{R}$ as basis vectors, leaving only the first-order terms in the parameter $S$, we obtain differential equations for the matrix elements of the density operator $\rho$ :

$$
\begin{gathered}
i \hbar \frac{\partial \rho_{L L}}{\partial t}=U\left(\rho_{R L}-\rho_{L R}\right)-\frac{i \hbar}{\tau_{L L}}\left(\rho_{L L}-\rho_{L L}^{0}\right), U \equiv U_{L R}^{L}=U_{L R}^{R}, \tau_{i j}=\tau_{j i}, i, j=L, R, \\
i \hbar \frac{\partial \rho_{R R}}{\partial t}=U\left(\rho_{L R}-\rho_{R L}\right)-\frac{i \hbar}{\tau_{L L}}\left(\rho_{R R}-\rho_{R R}^{0}\right), \rho_{L L}^{0}=\frac{1}{\left[1+\exp \left(\frac{E_{L}^{0}-E_{R}^{0}+2 B \varepsilon}{k T}\right)\right]}, \rho_{i \neq j}^{0}=0, \\
i \hbar \frac{\partial \rho_{L R}}{\partial t}=\left(\Delta-\frac{i \hbar}{\tau_{L R}}\right) \rho_{L R}-U\left(\rho_{L L}-\rho_{R R}\right), \rho_{R R}^{0}=\frac{\exp \left(\frac{E_{L}^{0}-E_{R}^{0}+2 B \varepsilon}{k T}\right)}{\left[1+\exp \left(\frac{E_{L}^{0}-E_{R}^{0}+2 B \varepsilon}{k T}\right)\right]}, \rho_{L R}=\rho_{R L}^{*}
\end{gathered}
$$

The diagonal matrix element $\rho_{L L}$ has the meaning of the probability of finding an atom in the left well. For simplicity, the system under study is characterized by two relaxation times: the longitudinal relaxation time of the system $\tau_{L L}=\tau_{R R}$ with an atom in the left (right) potential well and $\tau_{L R}=\tau_{R L}$ the transverse relaxation time of off-diagonal matrix elements.

When the time of the tunneling transition of an atom significantly exceeds the time of longitudinal or transverse relaxation, quantum coherence is violated. The probability of detecting a particle in a certain state can be found from the solution of the classical kinetic equations obtained by reducing the system Equations (5)-(7). The relaxation time has an exponential dependence on temperature $\omega_{0}^{-1} \exp \left(\frac{V}{k T}\right)$, where $V$ is the height of the 
interwell barrier. When the temperature is high (the relaxation time is short) and the shear deformation is less than the critical one $\varepsilon<\varepsilon_{*}, \varepsilon_{*}=-\frac{E_{L}^{0}-E_{R}^{0}}{2 B}$, the two-level system relaxes into a thermodynamically equilibrium state with $\rho_{L L}^{0}>\frac{1}{2}, \rho_{R R}^{0}<\frac{1}{2}$, in which the atom is in the left well with a higher probability than in the right well. When the deformation is equal to the critical $\varepsilon=\varepsilon_{*}$, the two-level system relaxes into the equilibrium state with $\rho_{L L}^{0}=\frac{1}{2}, \rho_{R R}^{0}=\frac{1}{2}$, in which the atom is with equal probability in the left and right wells. When the deformation is greater than the critical one $\varepsilon>\varepsilon_{*}$, the two-level system relaxes to the equilibrium state with $\rho_{L L}^{0}<\frac{1}{2}, \rho_{R R}^{0}>\frac{1}{2}$, in which the atom is in the right well with a greater probability than in the left well.

If the period of quantum oscillations is much shorter than the time of longitudinal and transverse relaxation, then the process of tunneling transition is coherent. The tunnel transition probability is an oscillating function of time, i.e., described by quantum kinetics. A linear combination of stationary wave functions $\Psi(x, t)$, satisfying the time-dependent Schrödinger equation with Hamiltonian (2) and the initial condition $\Psi(t=0)=\Psi_{L}$, makes it possible to find the probability of finding an atom in the left well:

$$
W(t)=a^{2}(t)=1-\frac{16[\Delta+\sqrt{D}]^{2} U^{2}}{\left([\Delta+\sqrt{D}]^{2}+4 U^{2}\right)^{2}} \sin ^{2}\left(\frac{\sqrt{D}}{2 \hbar} t\right), a(t)=\int d x \Psi(x, t) \Psi_{L}(x)
$$

The function $W(t)$ oscillates from unity to the minimum value, which is determined by the difference in energies in two states $\Delta$ and the magnitude of the product $U^{2}$. If $\Delta=0$, then $W(t)=1-\sin ^{2}\left(\frac{U}{\hbar} t\right)$. The probability of finding an atom in the left well varies from one to zero with a period $\tau=\frac{\pi \hbar}{U}$, and the atom spends the same time in both wells. If $\Delta^{2} \gg 4 U^{2}$, then $W(t)=1-4 \frac{U^{2}}{\Delta^{2}} \sin ^{2}\left(\frac{U}{\hbar} t\right)$. The atom is in the left potential well almost all of the time.

When the shear strain $\varepsilon$ is zero, the case $\Delta=\Delta_{0}=E_{L}^{0}-E_{R}^{0}<0, \Delta^{2} \gg 4 U^{2}$ is realized, and the atom is only in the left well. At a critical value of deformation $=\varepsilon_{*}$, the case $\Delta=0$ is reached and the atom tunnels into the right well. Further increase in deformation leads to the case $\Delta>0 \Delta^{2} \gg 4 U^{2}$, and the atom is only in the right well. The static displacement of an atom is an elementary act of structural relaxation of a medium under load and is accompanied by stress relaxation. This leads to a change in the positions of atoms from the nearest environment and "freezing" of the changed shape of the double-well potential. This process is reversible; after a slow removal of the load, the atom returns to the left well again. This is how the elementary inelastic reversible deformation proceeds in metallic glass at $T=0$.

Metallic glass contains a large number of relaxation centers containing two-level systems, which are described by a double-well potential, resonance detuning $\Delta$, and tunnel transition frequency $\omega_{K}$. Two groups of such double-well potentials can be distinguished-soft (with an interwell barrier $V \sim 10^{-4}-10^{-2} \mathrm{eV}$ ) and hard (with $V \sim 10^{-1}-1 \mathrm{eV}$ ) [12-14,24]. In the temperature range $T \cong 1-300 \mathrm{~K}$, thermal jumps between the states of a two-level system with a barrier $V \sim 10^{-4}-2.4 \cdot 10^{-2} \mathrm{eV}$ are activated. Therefore, the quantum tunneling mechanism is more probable for a two-level system with a hard potential.

Let us estimate the temperature $T_{*}$ at which the frequency of the quantum sub-barrier tunneling of an atom $\omega_{K}$ becomes equal to the frequency of the above-barrier fluctuation hopping $\omega_{T}$. Using the Gamow formula and Arrhenius's law, equating the exponents, we get

$$
\omega_{K}=\omega_{0} \exp \left(-\frac{a}{a_{d B}}\right), \omega_{T}=\omega_{0} \exp \left(-\frac{V}{k T}\right), a_{d B}=\frac{\hbar}{\sqrt{2 m V}}, T_{*}=\sqrt{\frac{V}{2 m}} \frac{\hbar}{a k}
$$

From (9), it can be seen that $T_{*}$ increases $\sqrt{V}$ proportionally and decreases $\sqrt{\frac{1}{m}}, \frac{1}{a}$ proportionally.

For a two-level system with a hard potential, the characteristic values are $V \cong 1 \mathrm{eV}, m \cong 10^{-25} \mathrm{~kg}$, and $a_{d B} \cong 0.56 \cdot 10^{-12} \mathrm{~m}$. With a barrier width $a=3 \cdot 10^{-11}$, $1.5 \cdot 10^{-11}, 0.9 \cdot 10^{-11} \mathrm{~m}(0.1,0.05,0.03$ interatomic distance), we obtain, respectively, 
$T_{*} \cong 65,216,721 \mathrm{~K}$ and the quantum tunneling frequency $\omega_{K} \cong 10^{-10} 20,100,000 \mathrm{~Hz}$. For local structural rearrangements with small displacements of atoms (much less than the interatomic distance), the tunneling effect is significant for room temperatures, and becomes the main at lower temperatures. Therefore, a possible physical mechanism of the inelastic deformation of metallic glasses (at room temperatures and below) is the quantum tunneling of some atoms in a double-well potential, or within an atomic group.

Under mechanical action on an amorphous material, a large number of two-level systems are excited, making a significant contribution to inelastic deformation and the formation of nanocrystals. The ability of amorphous metal alloys to deform irreversibly and to form nanocrystals is associated with the collectivized metallic character of the interatomic bond. In the presence of conduction electrons, irreversible processes of local collective atomic rearrangements can be carried out much more easily.

\section{Electron Localized States in Potential Nanometer Well and Phason Formation}

Let us assume that the electron moves in a three-dimensional spherically symmetric potential well having experienced the action of central force. In the simplest case, the potential energy $U(r)$ depends only on the distance between the electron and the force center $r$. Let the three-dimensional potential well have the form $U(r)=-U_{0}$ at $r \leq a$ and be equal to zero at $>a$. In a spherical coordinate system, the Schrödinger equation for stationary states has the form [26]

$$
\frac{1}{r^{2}} \frac{d}{d r}\left(r^{2} \frac{d}{d r} \Psi\right)+\frac{2 m}{\hbar^{2}}(E-U(r)) \Psi=0
$$

As solutions to Equation (10), which are finite at $r=0$ and vanish at $r \rightarrow \infty$, we can take:

$$
\Phi=\operatorname{Bsin}(\mathrm{kr}), \text { at } r \leq a, \Phi=\operatorname{Cexp}(-\alpha \mathrm{r}), \text { at } r>a, k=\sqrt{\frac{2 m\left(E+U_{0}\right)}{\hbar^{2}}}, \alpha=\sqrt{\frac{-2 m E}{\hbar^{2}}}
$$

Since we consider the particle inside a potential well, it is necessary to count $<0$. Thus, the problem of Equations (10) and (11) has been reduced to the problem of the motion of an electron in a one-dimensional potential well. Therefore, the energy levels are determined in the same way. The only difference now is in the necessity to discard states with even wave functions and leave only states with odd wave functions.

The fundamental difference between a one-dimensional potential well and a threedimensional one is that for a one-dimensional well there is at least one eigenvalue of energy with even wave function. In the case of a spherically symmetric rectangular well, this may not be the case. It can be seen from the relation $k^{2}+\alpha^{2}=\frac{2 m U_{0}}{\hbar^{2}}$ if $\frac{2 m U_{0}}{\hbar^{2}} a^{2}<\left(\frac{\pi}{2}\right)^{2}$, i.e.,

$$
U_{0}<\frac{\pi^{2} \hbar^{2}}{8 m a^{2}}
$$

that the curve given by the equation $\operatorname{tg}\left(\frac{k a}{2}\right)=-\frac{k}{\alpha}$ will never intersect the circle given by the equation $k^{2}+\alpha^{2}=\frac{2 m U_{0}}{\hbar^{2}}$. This means that when condition (12) is satisfied, not a single level of the discrete energy spectrum will appear in the potential well (due to the fact/the depth of the potential is not enough/the power of the well is too low).

Substituting the values of the fundamental constants $\hbar \cong 1 \cdot 10^{-34} \mathrm{~J} \cdot \mathrm{s}$, the electron mass $m \cong 9 \cdot 10^{-31} \mathrm{~kg}$, for the radii of the first, second, and third coordination sphere $a_{1}=0.35 \cdot 10^{-9} \mathrm{~m}, a_{2}=0.7 \cdot 10^{-9} \mathrm{~m}$, and $a_{3}=1.05 \cdot 10^{-9} \mathrm{~m}$, we obtain $U_{0}\left(a_{1}\right)=0.7 \mathrm{eV}$, $U_{0}\left(a_{2}\right)=0.18 \mathrm{eV}$, and $U_{0}\left(a_{3}\right)=0.08 \mathrm{eV}$, respectively. From physical considerations, it follows that the perfect local order can propagate starting from the first coordination sphere. The minimum radius of the potential well is equal to the interatomic distance $a_{1}=0.35 \cdot 10^{-9} \mathrm{~m}$. For a localized state of an electron to appear in a potential well with a minimum radius, the depth of the potential well of the resulting nanocrystal should be approximately $1 \mathrm{eV}$. 
At a high interfacial surface energy inherent in solid-phase transformation, the fluctuation formation of a region of a new crystalline phase leads to a significant change in the thermodynamic potential and may turn out to be unstable (nonequilibrium). However, in the presence of the attraction of a conduction electron in the formed region of a new crystalline phase, the electron can be localized in there. In this case, during the formation of a new region, if the decrease in the electron energy exceeds the growth of the thermodynamic potential energy of a new crystalline phase, then the fluctuation is stabilized. The arising thermodynamically stable formation of the new phase region with a localized electron in it (nonequilibrium in the absence of an electron) is called a phason [18].

In Section 1 of this work, it is shown that the formation of the nanoregion of the crystalline phase in an amorphous metal system can also occur by quantum tunneling stimulated by shear deformation. Therefore, the process of the collective atomic rearrangement of the relaxation center into a more ordered configuration (a transition between states in a two-level system, stimulated by shear deformation) can be accompanied by the localization of a conduction electron in the resulting potential well.

Let us consider a center of relaxation of radius $R$, which has experienced a shear deformation, to which the potential well with depth $-U$ corresponds to (with respect to the average potential energy of the amorphous state). During the transition between states in a two-level system, the change in the thermodynamic potential $\Delta \Phi$ is expressed by the formula

$$
\Delta \Phi(R)=\frac{4}{3} \pi R^{3} \varphi+4 \pi R^{2} \sigma_{s}+E_{e}(R), E_{e}(R)=-U+\frac{\hbar^{2} k^{2}}{2 m_{*}}
$$

where $E_{e}(R)$ is the energy of an electron in a potential well with a depth $-U$ and $m_{*}$ is the effective mass of an electron. Here, $\varphi<0$ is the difference between the densities of thermodynamic potentials of the new ordered state of atoms in the relaxation center and the initial disordered amorphous state, and $\sigma_{\mathcal{S}}$ is the interphase surface energy. Using the condition $2 R=\lambda=\frac{2 \pi}{k}$, where $\lambda$ is the wavelength of the electron, and substituting $k$ into the expression for the kinetic energy of the electron (13), we find $\frac{\hbar^{2} k^{2}}{2 m_{*}}=\frac{\hbar^{2} \pi^{2}}{2 m_{*} R^{2}}$.

$\Delta \Phi$ becomes negative (the deformed relaxation center is stable) at $U$, greater than a certain value $U_{0}$. The minimum value $\Delta \Phi$ corresponds to the critical value $R_{0}$, which determines the radius of the stably existing deformed relaxation center. Let us determine the value of $R_{0}$ and $U_{0}$. Equating $\frac{\partial \Delta \Phi(R)}{\partial R}$ to zero, for a large interfacial surface energy $|\varphi| R_{0} \ll 2 \sigma_{s}$, we obtain

$$
R_{0}=\left(\frac{\hbar^{2} \pi}{8 \sigma_{s} m_{*}}\right)^{\frac{1}{4}}, U_{0}=8 \pi \sigma_{s} R_{0}^{2}=(2 \pi)^{\frac{2}{3}} \sqrt{\frac{\hbar^{2} \sigma_{s}}{m_{*}}}
$$

For the value of $\sigma_{S}=20 \mathrm{erg} / \mathrm{cm}^{2}\left(1 \mathrm{erg}=10^{-7} \mathrm{~J} ; 1 \mathrm{~cm}=10^{-2} \mathrm{~m}\right)$, which is characteristic of metals [18], and the condition $|\varphi| R_{0} \ll 2 \sigma_{s}$, the critical radius of the deformed relaxation center is $R_{0} \cong 0.5 \mathrm{~nm}$, and the value $U_{0}$ is approximately $1 \mathrm{eV}$. It is clear from physical considerations that the minimum radius of a stable deformed relaxation center is approximately equal to the radius of the second coordination sphere. Since the theoretically estimated radius of a phason coincides in order of magnitude with the experimentally founded value of the radius of the nanocrystal [2-4,15], it is reasonable to assume that the possible mechanism for the nanocrystal formation in an amorphous NiTi metal film is the phason formation.

\section{Discussion: Physical Representation, Reasons and Conditions for Nanocrystal Formation, and Model Parameters Estimation}

In the framework of the model, the equations of the density operator are formulated, taking into account the relaxation term and the field of static shear deformation. The probabilities of the transitions of an atom in a double-well potential by thermal and 
athermal, i.e., quantum, mechanisms are determined. The obtained results allow us to formulate a physical idea of the nanocrystal formation and make conclusions about proper conditions for the crystallization.

At the initial moment of time, an amorphous metallic medium contains a large number of nanoregions (relaxation centers) as well as free volume and has a disordered atomic structure. The single action of infrasound (shear deformation) on the relaxation center, an atom (located in a double-well potential) or a group of atoms (which have two configurations with slightly different energies) provokes both thermal fluctuation (thermal mechanism) and quantum tunneling to new equilibrium positions (athermal mechanism). At a critical value of deformation $\varepsilon_{*}$ (corresponding to the material proportionality limit), the resonance detuning becomes zero and the atom tunnels. At room temperature, local structural rearrangement with small static displacements of atoms (much less than the interatomic distance) occurs with a tunneling frequency higher than the thermal hopping frequency. Thus, at room temperature and lower temperatures, the athermal (quantum) mechanism of inelastic deformation prevails. This conclusion is consistent with the experimental result that with an increase in the initial temperature of the sample (from 25 to $200{ }^{\circ} \mathrm{C}$ ), the distribution and size of crystalline clusters practically does not change [4]. As a result of inelastic deformation, a nanocluster with a crystalline atomic order appears at the relaxation center, but it may turn out to be unstable in the absence of infrasound.

Since the relaxation time and time of tunnel transition depend on temperature differently, both mechanisms (thermal and athermal) are realized upon the deformation of metallic glasses. With the decreasing of temperature, the thermal motion of atoms is getting to be frozen; as a consequence, the relaxation rate decreases exponentially accordingly to the Arrhenius law. Hence, at low temperatures, the rate of the tunneling transition exceeds the relaxation rate and coherent tunneling will occur. As the temperature rises, the relaxation rate increases faster than the tunneling rate. These values are initially compared, and then the relaxation rate can significantly exceed the tunneling rate. The transition process becomes thermofluctuational, and an ordered nanocluster arises due to thermal fluctuations. Thus, by changing the temperature, one can change the kinetics of the process of inelastic deformation of metallic glasses from quantum to classical.

The conditions for the appearance of a localized state of a conduction electron at the potential well of a nanocrystal are determined. The critical values of the potential well depth and the radius of a stable nanocrystal are calculated. Analysis of the obtained results allows us to formulate the following conclusions.

Under repeated exposure to infrasound, a nanocluster with a crystalline atomic order grows and reaches a critical radius. The nanocluster achieved the value of the critical radius and creates a local potential well in which the conduction electron appears in a bounded state. If the electron is attracted to the region of the unstable crystalline phase and is localized in there, then a decrease in the electron energy can be compensated for the surface energy of the interface. Thus, stable formation arises, also known as a phason (stable nanocrystal). The physical reason for the appearance of the stable nanocrystal is in the conduction electron localization by the potential well created by the nanocrystal.

The radius of the phason is determined by the value of the interfacial surface energy $\sigma_{s}$, and an increase $\sigma_{s}$ leads to a decrease in the radius of the phason. Physically, the radius of the phason cannot be less than the radius of the first coordination sphere. For the value $\sigma_{s}=20 \mathrm{erg} / \mathrm{cm}^{2}$, which is characteristic of metals [18], the critical radius of the phason is $R_{0} \cong 0.5 \mathrm{~nm}$. The critical value of the depth of the potential well $U_{0}$, i.e., the condition under which a phason with $R_{0} \cong 0.5 \mathrm{~nm}$ is formed, is approximately $1 \mathrm{eV}$.

Since the theoretical estimation of the critical radius of a phason coincides in order of magnitude with the experimentally founded value of the radius of a nanocrystal [2-4,15], it is reasonable to assume that the mechanism of the formation of a nanocrystal in a metallic glass is the mechanism of the phason formation. When conduction electrons are localized on nanocrystals formed as a result of inelastic deformation, they lower the energy of the 
system $[2,4]$. As a result, the state with nanocrystals becomes stable and a locally stable nanostructure appears (with respect to the globally stable crystal structure [3]).

It has been experimentally established $[2-4,15]$ that the morphology of nanocrystals depends on the method of nanocrystallization. The difference in the morphology of nanocrystals, isotropic upon annealing [15] and anisotropic upon exposure to infrasound [2-4], is associated with different mechanisms of nucleation and growth of nanocrystals. During nanocrystallization by high-temperature annealing, the nucleation and growth of nanocrystals are controlled by thermal fluctuation processes. However, at room temperature, the contribution of thermal fluctuation processes is small, inelastic deformation and nanocrystallization occur due to quantum transitions in nanoscale two-level systems, stimulated by infrasonic vibrations. The anisotropy of the nanocrystal shape is determined by the strain potential tensor, which depends on the local glass structure in the place where the two-level system is located.

Let us estimate the density of the stored potential energy of static displacements of atoms (inelastic deformation) under infrasonic action on the NiTi amorphous film. The dimensions of the film are known from the experiment [2-4]: length $l=1 \cdot 10^{-2} \mathrm{~m}$, width $r=1 \cdot 6 \cdot 10^{-3} \mathrm{~m}$, and thickness $d=4 \cdot 10^{-5} \mathrm{~m}$. The time of the cyclic mechanical action is $10 \mathrm{~min}$, and the frequency of the action is $20 \mathrm{~Hz}$. The amplitude of the tensile displacement of the infrasound is $A=4 \cdot 10^{-6} \mathrm{~m}$, and the relative longitudinal elongation of the film is $=\frac{A}{l}=4 \cdot 10^{-4}$. It is known that the potential energy density of homogeneous longitudinal elastic deformation is equal to $=\frac{E}{2} \cdot \varepsilon^{2}$, where $\mathrm{E}$ is the modulus of longitudinal elasticity (Young's modulus). The NiTi amorphous film is an alloy in which the main elements are nickel (45 percent) and titanium (41 percent). It is known that $E_{N i} \approx 220 \mathrm{GPa}$, $E_{T i} \approx 110 \mathrm{GPa}$, and their average value is equal to $E \approx 165 \mathrm{GPa}$. Taking into account the average value $E \approx 165 \mathrm{GPa}$ and the relative elongation $\varepsilon=4 \cdot 10^{-4}$, the potential energy density is equal to $e \approx 1.3 \cdot 10^{4} \mathrm{~J} / \mathrm{m}^{3}$.

Further, let us estimate the numerical value of the potential energy of uniform longitudinal elastic deformation per atom of the medium. Since the concentration of atoms in the medium is approximately equal to $0.9 \cdot 10^{29}$ atom $/ \mathrm{m}^{3}$, the energy per atom per one deformation cycle is equal to $e_{a} \approx 1 \cdot 10^{-6} \mathrm{eV} /$ atom. During the time of exposure to infrasound, $n=12,000$ cycles of mechanical deformation occur; therefore, the energy supplied to the system during the entire exposure time is $n \cdot e_{a} \approx 1.2 \cdot 10^{-2} \mathrm{eV} /$ atom. It is more probable that the small part (estimations are about 10 percent) of this energy can be converted into potential energy of inelastic deformation. Therefore, the value of the stored potential energy of inelastic deformation is $0.1 \cdot n \cdot e_{a} \approx 1.2 \cdot 10^{-3} \mathrm{eV} /$ atom, which is much less than the kinetic energy of an atom at room temperature $k T_{0} \approx 0.024 \mathrm{eV} /$ atom.

The latent heat of amorphous-crystalline transformation, determined through the temperature difference between amorphous and crystalline states $\Delta T \approx 150 \mathrm{~K}$ in the TiCu alloy [27], is $L_{a}=k \Delta T \approx 0.012 \mathrm{eV} /$ atom, which we can consider as an example. For the NiTi alloy, the latent heat of transformation is $L_{a} \approx 0.01 \mathrm{eV} /$ atom [4]. It has been experimentally established [2-4] that the latent heat of amorphous-crystalline transformation slightly decreases after 12,000 cycles of infrasonic exposure. Therefore, the free energy of the amorphous state with nanocrystals slightly decreases in comparison with the free energy of the amorphous state. It is reasonable to assume that the latent heat of transformation of an amorphous state into a nanostructured state (nanocrystals in an amorphous matrix) is much less than $L_{a} \approx 0.01 \mathrm{eV} /$ atom, for example, $0.1 \cdot L_{a} \approx 0.001 \mathrm{eV} /$ atom. Thus, the latent heat of transformation of an amorphous state into a nanostructural state $0.1 \cdot L_{a} \approx 0.001 \mathrm{eV} /$ atom coincides in order of magnitude with the value of the stored potential energy of inelastic deformation $0.1 \cdot n \cdot e_{a} \approx 1.2 \cdot 10^{-3} \mathrm{eV} /$ atom.

As was experimentally established in [4], when the frequency is below $5 \mathrm{~Hz}$ or the vibration amplitude is down to $1 \mu \mathrm{m}$, crystalline clusters do not appear in the amorphous matrix. Therefore, the following conclusion can be formulated: the occurrence of the instability of the amorphous state and following transformation to the nanostructured state is based on the accumulation of the potential energy of inelastic deformation to a 
critical value equal to the latent heat of the transformation of the amorphous state into the nanostructured state.

\section{Conclusions}

The relaxation centers including disordered nanoregions with two-level systems are the causes giving rise to the nanocrystal formation. When exposed to infrasound, the two-level systems, of which there are many, are excited, significantly contributing to inelastic deformation and the formation of nanocrystals. The physical mechanism of the nanocrystallization of metallic glass under mechanical action includes both local thermal fluctuations and the additional quantum tunneling of atoms stimulated by shear deformation. A crystalline nanocluster appears as a result of local atomic rearrangement growing increasingly exposed to infrasound. It is possibly unstable in the absence of infrasound. When the radius of the nanocluster reaches a critical value, the potential well appears. Bounding the conducting electron, it forms the phason. The estimated values (in case of the NiTi film) of the phason's radius and the depth of the nanometer potential well are $0.5 \mathrm{~nm}$ and $1 \mathrm{eV}$, respectively.

We consider the fact that the proposed microscopic mechanism makes it possible to formulate physical reasons and conditions for the appearance of nanocrystals, and we describe results and regularities obtained experimentally both quantitatively and qualitatively.

Funding: The work was performed according to the government research assignment for ISPMS SB RAS, project topic number FWRW-2019-0031.

Institutional Review Board Statement: Human or animal studies have not been conducted.

Informed Consent Statement: Human studies have not been conducted.

Data Availability Statement: All the necessary data confirming the obtained results are contained in the manuscript.

Conflicts of Interest: There is no conflict of interest.

\section{References}

1. Gunderov, D.; Astanin, V. Influence of HPT deformation on the structure and properties of amorphous alloys. Metals 2020, 10, 415. [CrossRef]

2. Belyaev, S.; Resnina, N.; Rubanik, V.; Shelyakov, A.; Niapomniashchay, V.; Ubyivovk, E.; Kasatkin, I. Influence of low-frequency vibrations on the structure of amorphous Ti40.7Hf9.5Ni44.8Cu5 alloy. Mater. Lett. 2017, 209, 231-234. [CrossRef]

3. Belyaev, S.; Rubanik, V.; Resnina, N.; Rubanik, V.; Ubyivovk, E.; Demidova, E.; Uzhekina, A.; Kasatkin, I.; Shelyakov, A. Crystallization of amorphous Ti40.7Hf9.5Ni41.8Cu8 alloy during the low-frequency mechanical vibrations at room temperature. Mater. Lett. 2020, 275, 128084-128087. [CrossRef]

4. Belyaev, S.; Rubanik, V.; Resnina, N.; Rubanik, V.; Ubyivovk, E.; Demidova, E.; Uzhekina, A.; Kasatkin, I.; Shelyakov, A. Variation in the structure of the amorphous NiTi-based alloys during mechanical vibrations. J. Non-Cryst. Solids 2020, $542,120101$. [CrossRef]

5. Christian, J.W. The Theory of Transformations in Metals and Alloys; Pergamon Press Ltd.: Oxford, UK, 1975.

6. Burton, W.K.; Cabrera, N.; Frank, F.C. The growth of crystals and the equilibrium structure of their surfaces. Philos. Trans. R. Soc. 1951, 243, 299-358. [CrossRef]

7. Köster, U.; Herold, U. Crystallization of Metallic Glasses. In Topics Applied Physics; Glassy Metals, I., Guntherrodt, H.-J., Beck, H., Eds.; Springer-Verlad: Berlin, Germany, 1981; pp. 225-259. [CrossRef]

8. Greer, A.L. Crystallisation kinetics of Fe80B20 glass. Acta Metall. 1982, 30, 171-192. [CrossRef]

9. Köster, U.; Meibhardt, J.; Birol, Y.; Aronin, A. Crystallization of Cu50Ti50 glasses and undercooled melts. Z. Metallkd. 1995, 86, 171-175. [CrossRef]

10. Anderson, P.W.; Halperin, B.I.; Varma, C.M. Anomalous low-temperature thermal properties of glasses and spin glasses. Philos. Mag. 1972, 25, 1-9. [CrossRef]

11. Phillips, W.A. Tunneling states in amorphous solids. J. Low Temp. Phys. 1972, 7, 351-360. [CrossRef]

12. Sussmann, J.A. Phonon Induced tunneling of ions in solids. Phys. kondens. Mater. 1964, 2, 146-160. [CrossRef]

13. Klinger, M.I. Low-temperature properties and localized electronic states of glasses. Sov. Phys. Usp. 1987, 30, 699-715. [CrossRef]

14. Slyadnikov, E.E. Pretransition state and structural transition in a deformed crystal. Phys. Solid State 2004, 46, 1095-1100. [CrossRef]

15. Luborsky, F.E. Amorphous Metallic Alloys; Butterworths: London, UK, 1983.

16. Peierls, R. Quantum Theory of Solids, 1st ed.; Clarendon Oxford Press: Oxford, UK, 1955. 
17. Mott, N.F.; Davis, E.A. Electronic Processes in Non-Crystalline Materials, 2nd ed.; Oxford at Clarendon Press: Oxford, UK, 1979.

18. Krivoglaz, M.A. Fluctuon states of electrons. Sov. Phys. Usp. 1974, 16, 856-877. [CrossRef]

19. Argon, A.S. Plastic-deformation in metallic glasses. Acta Metall. 1979, 27, 47-58. [CrossRef]

20. Taub, A.I.; Spaepen, F. The kinetics of structural relaxation of a metallic glass. Acta Metall. 1980, 28, 1781-1788. [CrossRef]

21. Gibbs, M.R.J.; Evetts, J.E.; Leake, J.A. Activation energy spectra and relaxation in amorphous materials. J. Mater. Sci. 1983, 18, 278-288. [CrossRef]

22. Kosilov, A.T.; Khonik, V.A. Directional structural relaxation and homogeneous flow of freshly quenched metallic glasses. In Proceedings of the Relaxation Phenomena in Solids, Seminar 'Relaksatsionnye Yavleniya v Tverdykh Telakh', Voronezh, Russia, 11 February 1993. Reference Number: AIX-25-051385; EDB-94-109615.

23. Egami, T.; Vitek, V. Local structural fluctuations and defects in metallic glasses. J. Non-Cryst. Solids 1984, 62, 499-510. [CrossRef]

24. Bakai, A.S. The polycluster concept of amorphous solids. In Glassy Metals III. Topics in Applied Physics; Springer: Berlin/Heidelberg, Germany, 1994; Volume 72, pp. 209-255. [CrossRef]

25. Pantel, R.; Puthof, G. Fundamentals of Quantum Electronics; Wiley: New York, NY, USA, 1969.

26. Landau, L.D.; Lifshitz, E.M. Quantum Mechanics. Non-Relativistic Theory, 3rd ed.; Pergamon Press Ltd.: London, UK, 1991.

27. Rogachev, A.S.; Vadchenko, S.G.; Shchukin, A.S.; Kovalev, I.D.; Aronin, A.S. Self-propagating crystallization waves in the TiCu amorphous alloy. JETP Lett. 2016, 104, 726-729. [CrossRef] 